\title{
Lymphocytic Adenohypophysitis and Neurohypophysitis
}

In recent years, lymphocytic hypophysitis has attracted considerable interest chiefly due to several impressive reports concerning the disease. The first case of lymphocytic hypophysitis was described in 1962 (1) and more than 100 cases similar to the original report have been reported $(2,3)$. Most of the cases affected were pregnant or immediately postpartum women who presented with visual symptoms, panhypopituitarism, and radiological appearance of a sellar mass mimicking pituitary adenoma.

See also p 293.

The histopathological examination shows chronic inflammation with lymphocytic infiltration and fibrosis in the anterior pituitary. The posterior lobe is histologically normal in most of the cases and diabetes insipidus before surgery is quite rare, suggesting that the disorder is "adenohypophysitis". Historically, adenohypophysitis has been described as lymphocytic hypophysitis, however the term hypophysitis may be somewhat ambiguous. The term "lymphocytic adenohypophysitis (LA)" seems to be correct in most of the cases. On the other hand, different types of lymphocytic hypophysitis have recently been reported. Imura et al proposed a new disease entity in 1993 (4) that they described as "lymphocytic infundibuloneurohypophysitis (LIN)". LIN has been characterized based upon the MRI appearance of thickening of the pituitary stalk and enlargement of the posterior lobe which lacks the hyperintense signal. Clinically, the patients present with diabetes insipidus; destruction of the anterior pituitary is not observed in all of the cases. Therefore, the lesion of LIN appears limited to the neurohypophysis including the pituitary stalk and/or posterior lobe. In this issue, Tsuji and colleagues (5) have reported a similar case of a male patient who presented with diabetes insipidus and a tumor-like lesion of the neurohypophysis. The histological examination revealed infiltration of lymphocytes in the posterior lobe. We agree that this case is a candidate for LIN and understand that LIN is different from LA in its anatomical extent of the lesion and subsequent symptoms. However, several interesting cases beyond this understanding have been reported. These cases have exhibited both dysfunction of adenohypophysis and neurohypophysis. Ahmed et al have reported two male cases who presented with panhypopituitarism and diabetes insipidus (6). They described that the lesion extended to involve the neurohypophysial system including the infundibulum, pituitary stalk, and posterior lobe, and termed these cases "necrotizing infundibulo-hypophysitis" based upon the histological features including necrosis in the entire pituitary. There have been more than 5 cases in the literature demonstrating dysfunction of the anterior pituitary and diabetes insipidus that have been reported as lymphocytic hypophysitis $(3,7)$. Nishioka et al have recently reported two cases of postmenopausal women, as a variant of LIN, demonstrating that inflammatory lesions were found in both the adenohypophysis and neurohypophysis (8). These reports raise the possibility that the inflammatory process may occur in both adeno- and neuro-hypophysial systems in an affected patient, although it is unclear which system is the principle site of inflammation. If the inflammatory invasion occurs in both systems, this disorder might be termed "lymphocytic adenoand neuro-hypophysitis". To establish this concept, we need more cases in which inflammation is histologically identified in both adeno- and neuro-hypophysis. Meanwhile, it is important to note that compression damage may be caused by the tumorous lesion other than the direct inflammatory invasion. In some cases of LA showing suprasellar extension of the tumorous lesion mimicking adenoma, neurohypophysial dysfunction might occur as is found in some cases of pituitary adenoma. The pathogenesis of LA is probably due to an autoimmunity of the pituitary gland, because several indications such as the detection of circulating antipituitary antibodies, the association with pregnancy, and the coexistence with autoimmune diseases highly support the autoimmune hypothesis $(2,3)$. Furthermore, experimental animal models of LA have been produced using pituitary tissue and an adjuvant (9). Is an autoimmune mechanism involved also in LIN? To date, we have not found any reports supporting the idea that LIN has an autoimmune basis. Only one case with Grave's disease has been described as LIN (4) and further studies including the coincidence of autoimmune disease and the detection of antipituitary antibodies by a highlyspecific method are required in LIN. Although the clinical management of LA, LIN, and variant cases has not been established, efforts to collect and analyze similar cases will provide further information.

Jiro TAKahara, MD and Makoto SATO, MD The First Department of Internal Medicine, Kagawa Medical University 1750 Miki-cho, Kida, Kagawa 761-07

\section{References}

1) Goudie RB, Pinkerton PH. Anterior hypophysitis and Hashimoto's disease in a young woman. J Pathol Bacteriol 83: 584, 1962.

2) Cosman F, Post KD, Holub DA, Wardlaw SL. Lymphocytic hypophysitis: Report of 3 new cases and review of the literature. Medicine 68: 240, 1989.

3) Thodou E, Asa SL, Kontogeorgos G, Kovacs K, Horvath E, Ezzat S. Clinical case seminar: Lymphocytic hypophysitis: Clinicopathological findings. J Clin Endocrinol Metab 80: 2302, 1995. 
4) Imura H, Nakao K, Shimatsu A, et al. Lymphocytic infundibuloneurohypophysitis as a cause of central diabetes insipidus. N Engl J Med 329: 683, 1993.

5) Tsujii S, Takeuchi J, Koh M, et al. A candidate case for lymphocytic infundibulo-neurohypophysitis mimicking a neurohypophysial tumor. Intern Med 36: 293, 1997.

6) Ahmed SR, Aiello DP, Page R, Hopper K, Towfighi J, Santen RJ. Necrotizing infundibulo-hypophysitis: A unique syndrome of diabetes insipidus and hypopituitarism. J Clin Endocrinol Metab 76: 1499, 1993.
7) Koshiyama H, Sato H, Yorita S, et al. Lymphocytic hypophysitis presenting with diabetes insipidus: Case report and literature review. Endocrine J 41: 93, 1994.

8) Nishioka H, Ito H, Sano T, Ito Y. Two cases of lymphocytic hypophysitis presenting with diabetes insipidus: A variant of lymphocytic infundibuloneurohypophysitis. Surg Neurol 46: 285, 1996.

9) Levine S. Allergic adenohypophysitis: New experimental disease of the pituitary gland. Science 158: 1190, 1967. 